\title{
Modélisation de l'action et contrefactuels. Un exemple exploratoire en didactique
}

\section{Gérard Sensevy et Nathalie Vigot}

\section{(2) OpenEdition \\ 1 Journals}

Édition électronique

URL : http://journals.openedition.org/trema/3536

DOI : 10.4000/trema.3536

ISSN : 2107-0997

\section{Éditeur}

Faculté d'Éducation de l'université de Montpellier

\section{Édition imprimée}

Date de publication : 1 octobre 2016

Pagination : 83-91

ISBN : 979-10-96627-01-1

ISSN : 1167-315X

\section{Référence électronique}

Gérard Sensevy et Nathalie Vigot, « Modélisation de l'action et contrefactuels. Un exemple exploratoire en didactique », Tréma [En ligne], 45 | 2016, mis en ligne le 01 janvier 2017, consulté le 07 mai 2019. URL : http://journals.openedition.org/trema/3536 ; DOI : 10.4000/trema.3536

Ce document a été généré automatiquement le 7 mai 2019.

Trema 


\title{
Modélisation de l'action et contrefactuels. Un exemple exploratoire en didactique
}

\author{
Gérard Sensevy et Nathalie Vigot
}

\section{Introduction}

1 Cet article se présente comme une contribution à l'étude du processus de modélisation en didactique $^{1}$. Dans une première partie, nous proposons une conception générale des modèles et de la modélisation, qui repose sur une certaine vision des rapports entre concret et abstrait. Une seconde partie décrit deux dialectiques entrelacées de l'action didactique, celle du connu (contrat) et de l'à connaître (milieu), et celle de l'expression et de la réticence, qui nous semblent cruciales pour l'étude et la compréhension de cette action. Dans la troisième partie du texte, nous proposons une notion, celle de stratégie contrefactuelle, dont nous tentons de montrer un usage pour le travail de modélisation, dans l'exploration des accomplissements pratiques. Une quatrième partie présente un travail empirique élaboré à partir de ces modèles.

2 Un tel projet s'inscrit, de manière exploratoire (Ligozat, Coquidé, Marlot, et Sensevy, 2014), dans une épistémologie qui voit la didactique comme la science du didactique (Chevallard, sous presse).

\section{Une conception générale de ce qu'est un modèle}

Dans cette première section, nous considérons le travail de production de modèles dans une relation particulière construite entre abstrait et concret. 


\section{1. Le modèle : un voir-comme pour agir dans la réalité}

4 Un modèle peut être conçu comme un voir-comme (Wittgenstein, 2004). Face à une réalité donnée, on considère seulement certains aspects de cette réalité et on met en relation ces différents aspects pour agir. On voit alors cette réalité comme un système fondé sur ces relations. Un autre voir-comme permettra de voir la réalité d'une autre manière, comme on peut voir tour à tour, selon l'exemple célèbre du canard-lapin utilisé par Wittgenstein, la même image comme représentant un lapin ou un canard:

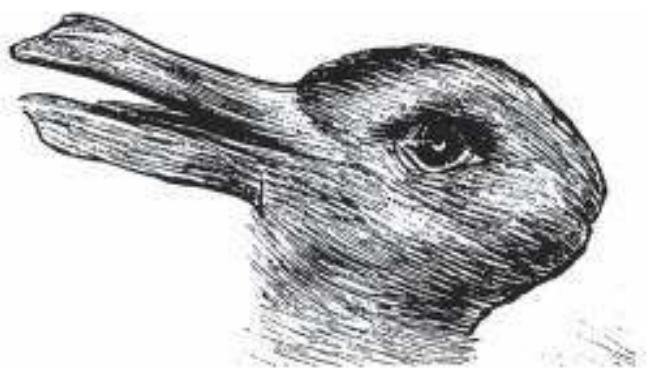

\section{Le canard-lapin Wittgensteinien}

5 Par exemple lorsque, pour entretenir mon jardin potager, je dois arracher des "mauvaises herbes", je vois la réalité comme construite avec des mauvaises herbes, à côté de salades qui ne sont pas de mauvaises herbes : je vais donc arracher telle herbe et ne pas toucher les salades. Bien entendu, les « mauvaises » herbes ne sont pas des mauvaises herbes en soi. Pour le pratiquant d'un autre type d'agriculture (cf. par exemple Fukuoka, 2005), elles pourront être complètement bénéfiques et leur présence activera donc un autre voir-comme. On saisit que, dans le cas ci-dessus, l'opération de modélisation est d'une part étroitement liée à l'action : je vois le réel d'une certaine manière pour réaliser une action. D'autre part, cette modélisation a été forcément apprise: quelqu'un m'a enseigné comment faire la différence entre les mauvaises herbes et la salade. Je vois la réalité avec les lunettes de mon action, telles qu'un « connaisseur » de cette action me les a transmises, m'a permis de les façonner.

6 La modélisation, au plan épistémologique, est donc une opération de réduction du réel, au sens où seules certaines de ses propriétés, de ses affordances, aurait dit Gibson (2014), sont prises en compte. Sa formulation dans un langage suppose une mise en référence, une particularisation, sans laquelle son usage est impossible. L'expression «il faut arracher les mauvaises herbes » est en elle-même une abstraction, qui, pour devenir opérationnelle, suppose un système de connaissances reliées à une certaine réalité concrète.

7 Il est nécessaire, selon nous, de généraliser cette vision pour comprendre l'opération de théorisation, de modélisation de la réalité. Modéliser une réalité, c'est toujours mettre en relation le concret d'un réel particulier et une abstraction qui permet de s'orienter dans ce réel, de le « voir comme », à certaines fins.

8 Mais que peut signifier le terme "concret " pour les sciences de la culture? Il désigne selon nous le concret de la praxis, le concret des accomplissements pratiques (Garfinkel, 2007). Ce concret exprime donc la logique de la pratique (Bourdieu, 1980), sa grammaire (Wittgenstein, 2004). L'un des enjeux majeurs des sciences de la culture est de 
comprendre cette grammaire endogène, immanente, à la pratique, sans l'arraisonner au moyen d'abstractions qui lui sont étrangères.

\section{2. L'ascension de l'abstrait au concret}

9 Une telle conception peut renverser radicalement les rapports classiquement établis entre l'abstrait et le concret, où la « production de l'abstrait » est pensée comme l'objectif final d'une démarche intellectuelle fondée sur l'identification de ce qu'un certain nombre d'éléments concrets possèdent en commun. Elle retrouve, selon nous, une vision marxienne des rapports du concret et de l'abstrait, dans le sens où l'activité scientifique organise l'ascension de l'abstrait au concret (Marx, 2009; Kosic, 1988; Illenkov, 1982; Engeström, Nummijoki, et Sannino, 2012). Nous pouvons décrire cette ascension comme suit.

Devant une réalité particulière, l'analyste commence d'abord par en abstraire une première forme de logique, un premier modèle. Il exprime cette logique en produisant ce que l'on pourrait appeler, en suivant Deleuze (1986), une «formule abstraite ». Pour ce qui concerne les sciences de la culture, on pourrait dire que cette formule abstraite, comme modèle, constitue une manière de décrire le sens pratique des agents, leur sens du jeu et donc d'effectuer une "première reconnaissance» de la logique (de la) pratique. Mais ce modèle, tel quel, ne rend pas justice à la complexité du concret : l'ascension de l'abstrait au concret consiste donc à "plonger» cette formule abstraite au sein de différents concrets, de différents particuliers, qu'on pourra considérer comme des exemples exemplaires (Kuhn, 1990), pour lui donner peu à peu une référence de plus en plus variée. L'exhibition de la logique pratique est alors conçue non pas comme la seule déduction d'un modèle en tant que formule abstraite, mais dans la production d'un $\mathrm{n}$ uplet du type (modèle M1, exemple 1, exemple 2, etc.).

Dans ce qui suit, nous allons donc procéder en trois temps : dans un premier temps (III.), nous allons produire un premier modèle, établi à partir d'une théorisation précédemment élaborée (Sensevy, 2011), qui tente d'appréhender l'action didactique selon deux dialectiques imbriquées. Dans un second temps (IV.), nous proposerons un second modèle (la notion de contrefactuels) dont l'une des fonctions principales tient précisément dans le processus de concrétisation des « formules abstraites » que constituent les modèles. Dans un troisième temps (V.), nous proposerons un exemple empirique de l'usage contrefactuel du modèle des « deux dialectiques ».

\section{Un modèle de l'action didactique : deux dialectiques entrelacées}

Sous une certaine description, le Professeur doit travailler le problème suivant: il est confronté à l'Élève, qui sait déjà certaines choses, qu'on peut décrire comme un déjà-là, en grande partie issu des relations précédentes que le Professeur et l'Élève ont construites autour du savoir, comme le modélise la notion de contrat didactique (Brousseau, 1998; Sensevy, 2011). Mais ce déjà-là n'a de sens, comme Brousseau l'a explicité, qu'en tant qu'équilibre provisoire destiné à être rompu. En effet, sur l'arrièreplan de ce contrat, le Professeur et l'Élève vont devoir aborder ce qui est à apprendre. 
13 La grammaire du jeu professoral comporte donc deux dimensions dialectiques, que nous caractérisons ci-dessous à partir d'éléments de la théorie de l'action conjointe en didactique, éléments que nous avons réorganisés pour tenter de mieux rendre compte de la dynamique de l'action.

14 La première dimension dialectique renvoie à la nature de l'information que le professeur apporte à l'élève pour que celui-ci résolve le problème. Cette action se situe entre deux pôles. Le premier renvoie au fait qu'il peut s'appuyer sur le déjà-là, par exemple en disant: «ceci, tu l'as déjà fait hier, c'est la même chose qu'hier ». Une description générale de ce genre d'action peut consister à dire que le professeur va aider l'élève à reconnaitre quelque chose qu'il a déjà rencontré, qui appartient à l'arrière-plan lié au problème abordé.

Le second pôle de l'action professorale consiste à orienter l'élève vers la mise en relation d'éléments du problème, qui lui permettront de le constituer comme tel pour le résoudre, par exemple en disant : « tu as ceci, tu as aussi cela, donc?».

16 Le professeur peut donc faire reconnaître le connu (premier pôle) ou aider l'élève à s'orienter dans l'inconnu (deuxième pôle). Connu et inconnu sont bien entendu relatifs et un gradient s'établit, au plan stratégique, entre les deux actions idéaltypiques d'appui sur le contrat (connu) et d'exploration des relations dans le milieu (inconnu, à connaître). Le professeur agit donc soit en direction de ce qui est déjà connu, soit en direction de ce qui est à connaître. C'est la dialectique du connu (contrat) et de l'à-connaître (milieu).

Cette première dimension dialectique de l'action professorale est nécessairement entrelacée dans une autre. Pour faire vivre cette dialectique du connu et de l'à-connaître, le professeur peut agir en disant/montrant certains aspects du problème (expression) et en taisant/cachant certains autres (réticence). C'est la dialectique de l'expression et de la réticence. Plus précisément, on pourrait dire que toute expression du professeur comporte une réticence, que toute réticence suppose une expression.

Par entrelacement de ces deux dialectiques, nous entendons le fait que le professeur peut s'exprimer (dire/montrer) ou être réticent (taire/cacher), dans le sens de la reconnaissance de choses connues ou dans celui de la mise en relation de choses à connaître.

19 L'entrelacement permet ici de décrire comment le problème se construit peu à peu dans la transaction professeur-élève et comment l'action (plus ou moins) conjointe permet d'avancer. C'est le processus de mésogénèse, qui fait passer l'élève d'une première reconnaissance d'éléments du problème à la mise en relation systémique de ces éléments.

La description dynamique de ce système stratégique requiert par ailleurs un descripteur de la part épistémique respective prise par chaque transactant dans la transaction ( topogénèse) et un descripteur de la manière dont le temps avance dans la transaction ( chronogénèse $e^{2}$.

\section{Modélisation et exploration du concret : les contrefactuels}

21 L'entrelacement des deux dialectiques (connu-à connaître/expression-réticence) de l'action professorale, tel que nous venons de l'expliciter ci-dessus, constitue une première formule abstraite destinée à être plongée dans le concret, pour en permettre 
l'exploration. Une telle structure devra décrire la logique immanente à la pratique. Mais elle permettra également d'envisager d'autres possibilités pratiques, sous la forme de stratégies contrefactuelles, souvent en se dégageant des fausses nécessités (Unger, 2007) liées à certaines approches de la pratique.

Devant une praxis didactique, donc, on pourra faire vivre un double mouvement. Décrire la pratique effectivement réalisée, par exemple à l'aide de l'entrelacement dialectique précisé ci-dessus, pour élucider la logique de l'action ; imaginer d'autres possibles, pour produire une stratégie contrefactuelle (CF), puis une autre, et encore une autre, etc. dont la description investira de différentes manière ledit entrelacement dialectique. Ces stratégies contrefactuelles (dont l'une sera la stratégie effectivement réalisée) permettent une exploration de la pratique concrète. Au-delà des significations en général attribuées à ce terme (contrefactuel ${ }^{3}$ ), il faut saisir que l'idée de contrefactuel est consubstantielle, dans une théorie de l'action, à l'agentivité, puisqu'elle peut rendre compte de la manière dont, au sein des accomplissements pratiques, les humains produisent des intentions et des choix, c'est-à-dire des stratégies. Comme nous l'avons précisé dans la première et la deuxième partie de cet article, l'usage de contrefactuels a également une fonction épistémologique. Il concrétise la "formule abstraite " que constitue le modèle, en montrant quels types de pratiques il permet de caractériser ou d'imaginer, et peut donc permettre en retour une accommodation dudit modèle. Un tel usage peut être vu comme une conceptualisation théorique visant à distinguer, comme le fait Yves Clot (2008) dans une inspiration vygotskienne, le réel de l'activité et l'activité réalisée.

\section{Analyser les stratégies réalisées et contrefactuelles : un exemple emblématique}

Pour avancer dans le travail d'analyse, nous allons maintenant nous centrer sur un exemple de pratique, qui nous semble pouvoir fournir un emblème de cet entrelacement des dialectiques que nous avons tenté de décrire ci-dessus. Avant d'entrer dans l'analyse proprement dite, rappelons qu'elle est le fruit, comme le reste de l'article, de deux auteurs, dont l'un est aussi le professeur qui a mis en œuvre la séance. Cette étude ne doit donc pas être prise comme l'analyse d'un chercheur sur la pratique d'un professeur. D'une part, elle est le fruit d'un travail conjoint accompli au sein d'une ingénierie coopérative (Sensevy, Forest, Quilio, et Morales, 2013 ; Sensevy, à paraître), dans lequel chercheur et professeur co-élaborent des analyses empiriques, à partir de conceptions rendues communes par une longue expérience partagée de la recherche. D'autre part, le professeur qui a mis en œuvre la séance analysée est lui-même également chercheur (Vigot, 2014).

Dans cette perspective, l'analyse des énoncés et des comportements proposée ci-dessous est bien l'expression d'un voir-comme, qui n'appréhende pas en soi une hypothétique réalité, mais qui la fait percevoir à partir des modèles que nous proposons.

\section{1. L'analyse du système stratégique réalisé}

Il s'agit d'un court épisode qui se déroule en lecture au Cours Préparatoire, au mois de décembre, donc à un moment où les élèves ignorent encore la signification de nombreux phonèmes. Nous sommes en début de séance. 
Le professeur $(\mathrm{P})$ vient de faire travailler les élèves sur le texte suivant : « Le père Noël va dans toutes les villes et villages ».

Le professeur s'apprête à continuer la lecture du texte, et demande aux élèves si quelqu'un connaît encore quelque chose dans le texte :

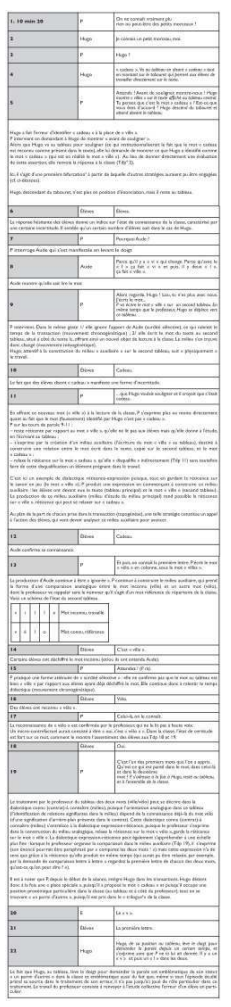

28 L'analyse qui précède a abouti à la reconnaissance d'un système stratégique réalisé, effectif. Mais on peut soutenir qu'il est possible d'aller plus loin dans l'étude pratique de cette action, en dégageant contrefactuellement ce qu'elle aurait pu être, non seulement avec un autre professeur en d'autres circonstances légèrement différentes (par exemple un peu plus tard ou un peu plus tôt dans l'année), mais par le même professeur dont l'activité se serait orientée dans d'autres embranchements de la pratique.

\section{2. L'analyse d'autres systèmes stratégiques contrefactuels}

29 Nous distinguons dans l'analyse contrefactuelle quatre stratégies, la cinquième étant la stratégie effectivement produite. Cette conceptualisation repose, comme nous l'avons avancé ci-dessus, sur des bifurcations de la pratique mais elle est en partie arbitraire : son usage n'est pas de "dire» ce qui aurait pu se passer mais, en imaginant d'autres possibles, de mieux comprendre le feuilletage de l'action, en saisissant mieux comment l'effectif s'est accompli à côté de, voire contre certaines autres options possibles.

\section{Stratégie contrefactuelle 1}

P estime que l'état épistémique de la classe est suffisant pour permettre la rectification immédiate. Il corrige donc l'erreur de Hugo en s'appuyant sur ce qu'il pense être un déjàlà (par exemple le fait que « ville » comporte un « $\mathrm{i}$ » et « cadeau » un « $\mathrm{a}$ »), en donnant la 
réponse lui-même. Par exemple, il dit directement à Hugo et à la classe : «ce n'est pas "cadeau", on entend un "a" dans cadeau, et il n'y a pas de "a" dans ville».

Dans ce contrefactuel, P s'appuie donc sur le connu, le déjà-là, le contrat didactique, auquel il attribue des propriétés qui permettront aux élèves de suivre son raisonnement. Son expression comporte peu de réticence, puisqu'il pense qu'il n'y a que peu de choses à découvrir.

$\mathrm{Au}$ plan topogénétique, la position du professeur est très haute (il apporte tout le savoir), celle des élèves est basse (ils suivent).

La chronogénèse est très rapide: peu de temps d'étude est donné aux élèves pour s'approprier les paroles du professeur, puisque celui-ci postule une distance épistémique faible entre l'état des élèves et ce qui leur est demandé.

\section{Stratégie contrefactuelle 2}

Dans cette stratégie, P facilite l'exploration du mot «ville». Pour cela, il demande aux élèves directement la première lettre du mot cherché. Cette expression consiste à organiser l'exploration du mot connu, un peu comme dans la stratégie effectivement réalisée, et suppose donc, contrairement à la première stratégie contrefactuelle ci-dessus, une certaine réticence motivée par l'exploration de l'à-connaître. Toutefois, par rapport à la stratégie effectivement réalisée, cette exploration prend ici la forme d'un questionnement qui peut ramener directement l'élève au connu.

$\mathrm{Au}$ plan topogénétique, la position du professeur est haute, dans le sens où il assume la responsabilité de fixer l'attention des élèves sur la première lettre, mais la topogenèse est plus partagée que dans la stratégie précédente, puisque les élèves ont la responsabilité de chercher par eux-mêmes la lettre initiale, et non pas seulement d'enregistrer le raisonnement professoral.

36 La recherche des élèves ralentit légèrement la chronogénèse par rapport à l'exemple précédent.

\section{Stratégie contrefactuelle 4}

37 Dans cette stratégie, $\mathrm{P}$ organise l'action des élèves en focalisant leur attention sur l'inconnu. Il écrit au tableau le mot «cadeau » et demande de l'étudier, en focalisant l'attention plus étroitement sur le mot, l'étude du décodage du mot en phonèmes (ce qui rend cette stratégie raisonnable, c'est que la lettre «a » est connue des élèves, et fournit donc un point connu dans l'à-connaître). Il s'agit d'une stratégie de réfutation, dans laquelle le professeur attend des élèves qu'ils produisent de leur propre mouvement le raisonnement qu'il tient lui-même dans la première stratégie contrefactuelle ci-dessus. L'expression du professeur contient la réticence de ce raisonnement (il écrit simplement le mot au tableau en demandant son étude, sans donner le raisonnement).

$\mathrm{Au}$ plan topogénétique, les élèves ont donc une responsabilité accrue par rapport aux stratégies précédentes, puisque la production du raisonnement de réfutation est à leur charge.

39 Au plan chronogénétique, cette stratégie suppose du temps donné aux élèves pour travailler le mot « cadeau » et pouvoir l'invalider face à « ville». 


\section{Stratégie contrefactuelle 5}

\begin{tabular}{|c|c|c|c|c|}
\hline \multicolumn{4}{|l|}{ Connu (contrat) } & \multirow{2}{*}{$\begin{array}{l}\begin{array}{l}\text { A connaître } \\
\text { (milieu) }\end{array} \\
\text { Stratégie CF } 5\end{array}$} \\
\hline Stratégie CF I & Stratégie CF 2 & Stratégie CF 3 & Stratégie CF 4 & \\
\hline $\begin{array}{l}\text { Dire directement : } \\
\text { «pas cadeau, ville » }\end{array}$ & $\begin{array}{l}\text { Demander aux } \\
\text { élèves la première } \\
\text { lettre du mot } \\
\text { « ville » }\end{array}$ & $\begin{array}{l}\text { Produire I } \\
\text { tableau « ville- } \\
\text { vélo » }\end{array}$ & $\begin{array}{l}\text { Ecrire « cadeau » } \\
\text { au tableau }\end{array}$ & $\begin{array}{l}\text { Laisser réfléchir } \\
\text { les élèves devant } \\
\text { le texte }\end{array}$ \\
\hline $\begin{array}{l}\text { Reconnaissance } \\
\text { immédiate du } \\
\text { connu (contrat) }\end{array}$ & $\begin{array}{l}\text { Reconnaissance du } \\
\text { connu (contrat) }\end{array}$ & $\begin{array}{l}\text { Travail connu/à- } \\
\text { connaître } \\
\text { (contrat-milieu) }\end{array}$ & \multirow{2}{*}{$\begin{array}{l}\text { Exploration de } \\
\text { l'à-connaître (mi- } \\
\text { lieu) (appui inféré } \\
\text { sur le « a » de } \\
\text { " cadeau ») }\end{array}$} & \multirow{2}{*}{$\begin{array}{l}\text { Exploration } \\
\text { «libre » de } \\
\text { l'à-connaître } \\
\text { (milieu) }\end{array}$} \\
\hline & & & & \\
\hline Top $P \gg E$ & Top P > E & Top $P=E$ & Top $P<E$ & Top $P \ll<E$ \\
\hline Chron ++ & Chron + & Chron $=$ & Chron - & Chron -- \\
\hline
\end{tabular}

\section{3. Le réalisé, le contrefactuel, quelques remarques synthétiques}

La dernière stratégie contrefactuelle consiste à penser que les élèves peuvent d'euxmêmes réfuter la production de « cadeau » et/ou faire la lecture de " ville ».

Le professeur se retire donc symboliquement de la scène didactique, en disant par exemple : «Alors Hugo dit que ce mot (en désignant « cadeau ») est le mot « ville ». Qu'en pensez-vous?». Ainsi, le professeur produit une réticence forte à la fois sur le connu (il ne renvoie à aucun mot du répertoire de la classe qui puisse faciliter l'enquête des élèves) et sur l'à-connaître, qu'il ne cherche pas à mettre en relation avec le connu.

Au plan topogénétique, la part épistémique prise par les élèves devient très importante.

Le mouvement chronogénétique appelé par cette stratégie ralentit considérablement le temps didactique.

\section{La stratégie réalisée (3)}

Les stratégies contrefactuelles rapidement décrites ci-dessus permettent de mieux saisir, par contraste, la structure et le dynamisme propre de la stratégie effectivement produite. On peut avancer que celle-ci se situe dans un entre-deux, à la fois aux plans des dialectiques entrelacées connu-à connaître/expression-réticence, au plan topogénétique (les responsabilités sont ici partagées entre professeur et élèves), et au plan chronogénétique (l'avancée du temps suppose des surdités sélectives à certaines productions d'élève, des ralentissements, des accélérations).

L'ensemble des stratégies étudiées ci-dessus peut s'appréhender dans le tableau synoptique suivant :

V. 3. Le realise, le contrefactuel, quelques remarques synthétiques

«L'exploration du concret » proposée ci-dessus, qui est aussi simultanément « le travail d'un modèle », pourrait être bien entendu considérablement détaillée et approfondie. De notre point de vue, une telle investigation doit s'établir au sein du programme de recherche d'un collectif instrumenté. Sans poser ici ne serait-ce que les linéaments d'un tel programme, on précisera simplement que ce travail du modèle, dès lors qu'on l'arrime de façon immanente à un concret particulier, nécessite par exemple d'intégrer des 
éléments génériques (qu'est-ce que le déchiffrage en lecture au début de $\mathrm{CP}$ ? Quelles sont les contraintes de l'apprentissage en collectif ?) et des éléments spécifiques à la classe (ce que ces élèves-là savent faire, ce que la biographie didactique de Hugo nous apprend) qui surdéterminent le jeu du professeur au plan temporel (chronogénèse) et donc au plan de la constitution du milieu (mésogénèse). Autrement dit, le travail du modèle demande à la fois une double avancée qu'on pourrait dire grammaticale (la logique de la pratique étudiée) et indexicale (la prise en compte de la singularité des lieux, des moments, des biographies individuelles et collectives).

\section{Pour conclure}

Dans cette étude, nous avons tenté de donner à voir une opération de modélisation qui accorde un primat épistémologique, théorique et méthodologique au concret de la praxis. Après avoir explicité une conception des modèles et de la modélisation fondée sur l'ascension de l'abstrait au concret, nous avons proposé deux modèles : un modèle générique de l'action didactique, celui de l'entrelacement des dialectiques du contrat et du milieu, et de la réticence et de l'expression; un modèle générique de l'exploration de la pratique dans ses possibles, celui des stratégies contrefactuelles. Nous avons amené au concret ces modèles dans un exemple empirique, que nous considérons comme le germe d'un « double » exemple emblématique. Un exemple emblématique de l'usage de chacun de ces deux modèles, d'une part ; un exemple emblématique de l'entrelacement des dialectiques par lequel on peut appréhender les possibles de l'action didactique, d'autre part.

Dans cette perspective, au-delà de leur éventuel intérêt scientifique, les modèles proposés dans cet article pourraient jouer un rôle dans la formation des professeurs et des chercheurs, et dans leur travail coopératif. Ils pourraient en effet contribuer à la fois à mieux faire saisir certains déterminants de la pratique et à ouvrir l'imagination de ses possibles.

Ceci suppose en particulier de tirer toutes les conséquences du primat de la praxis assumé dans cet article, en engageant un travail collectif d'analyse de l'action didactique conjointe, des conceptions, des engagements, des valeurs et des normes qui la traversent - tant du côté du professeur que de celui des élèves. Il s'agirait alors de rendre explicite ce qui donne forme aux stratégies qui font la pratique.

\section{BIBLIOGRAPHIE}

Bourdieu, P. (1980). Le sens pratique. Paris : Éditions de Minuit.

Brousseau, G. (1998). Théorie des situations didactiques. Grenoble : La pensée sauvage.

Chevallard, Y. (sous presse). La TAD et son devenir : rappels, reprises, avancées. In G. Cirade et al. (Éds), Évolutions contemporaines du rapport aux mathématiques et aux autres savoirs à l'école et dans la société. Actes du 4e congrès international sur la TAD. 
Clot, Y. (2008). Travail et pouvoir d'agir. Paris : PUF.

Deleuze, G. (1986). Foucault. Paris : Minuit.

Engeström, Y., Nummijoki, J., and Sannino, A. (2012). Embodied Germ Cell at Work: Building an Expansive Concept of Physical Mobility in Home Care. Mind, Culture, and Activity, 19(3), 287-309.

Fukuoka, M. (2005). La Révolution d'un seul brin de paille: Une introduction à l'agriculture sauvage. Paris : Guy Trédaniel Éditeur.

Garfinkel, H. (2007). Recherches en ethnométhodologie. Paris : P.U.F.

Gibson, J. J. (2014). Approche écologique de la perception visuelle. Bellevaux : Éditions Dehors.

Ilyenkov, E. (1982). The Dialectics of the Abstract and the Concrete in Marx's Capital. Moscow: Progress Publishers.

Kosík, K. (1988). La Dialectique du concret. Paris : Éditions de la Passion.

Kuhn, T. (1990). La tension essentielle : tradition et changement dans les sciences. Paris : Gallimard.

Ligozat, F., Coquidé, M., Marlot, C. et Sensevy, G. (2014). Didactiques et/ou didactique. Poursuivre le travail de problématisation. Éducation et didactique, 8(1), 101-115.

Margolinas, C. (2005). Les bifurcations didactiques : un phénomène révélé par l'analyse de la structuration du milieu. In A. Mercier et $\mathrm{C}$. Margolinas, Balises en didactique des mathématiques (pp.1-12). Grenoble : La pensée sauvage.

Marx, K. (2009). Le Capital : critique de l'économie politique : quatrième édition allemande. Paris : P.U.F.

Sensevy, G. (1998). Institutions didactiques. Paris : P.U.F.

Sensevy, G. (2011). Le sens du savoir. Éléments pour une théorie de l'action conjointe en didactique. Bruxelles : De Boeck.

Sensevy, G., Forest, D., Quilio, S. and Morales, G. (2013). Cooperative engineering as a specific design-based research. ZDM, The International Journal on Mathematics Education, 45(7), 1031-1043.

Sensevy, G. (à paraître). Le collectif en didactique. Quelques remarques. Conférence à la 18e école d'été de didactique des mathématiques. Brest, Août 2015.

Schubauer-Leoni, M.-L. (1997). Interactions didactiques et interactions sociales : quels phénomènes et quelles constructions conceptuelles ? Skolé, 7, 103-134.

Unger, R. M. (2007). The self awakened: pragmatism unbound. Cambridge: Harvard University Press.

Vigot, N. (2014). Temps des pratiques de savoir, dispositifs et stratégies professorales : une étude de cas en mathématique au cours préparatoire : Journal du Nombre et Anticipation. (Thèse en Sciences de l'Éducation). Brest : Université de Brest.

Wittgenstein, L. (2004). Recherches philosophiques. Paris : Gallimard. Margolinas, C. (2005). Les bifurcations didactiques : un phénomène révélé par l'analyse de la structuration du milieu. In $\mathrm{A}$. Mercier et C. Margolinas, Balises en didactique des mathématiques (pp.1-12). Grenoble : La pensée sauvage.

\section{NOTES}

1. Des deux chercheurs co-auteurs de cet article, l'une, Nathalie Vigot, est le professeur dont la pratique est étudiée. 
2. Certains dispositifs peuvent par ailleurs être considérés en soi comme des dispositifs chronogénétiques. Voir par exemple Sensevy (1998) et Vigot (2014).

3. Il ne nous est pas possible, dans le cadre nécessairement limité de cet article, de recenser les différentes sémantiques du terme « contrefactuel » dans les différentes sciences.

4. Tdp pour « Tour de parole».

5. Le terme " bifurcation " peut ici être mis en lien avec la proposition théorique élaborée par Claire Margolinas (2005). Une stratégie contrefactuelle peut être en effet conçue (non exclusivement) comme une enquête menée à partir d'un point de bifurcation.

6. L'idée de «trilogue », en didactique, a été proposée par M.-L. Schubauer-Leoni (1997).

\section{RÉSUMÉS}

Cet article contribue à l'étude de la modélisation en didactique. Dans la conception proposée ici, la modélisation a pour fonction d'organiser l'ascension de l'abstrait au concret. Puis il décrit un modèle de l'action didactique, qui entrelace deux dialectiques de cette action. Un second modèle, celui de contrefactuel, dont est postulé un usage pour la modélisation de la pratique, est ensuite explicité. L'article présente enfin un travail empirique exemplifiant ces modèles.

This paper aims to contribute to the study of modeling in didactics. We first propose a general conception of modeling, focused on the ascent from abstract to concrete. Then we describe a model of didactic action, which entangles to dialectics of this action. After that, we propose a counterfactual model, which can play a specific role in the modeling process. We exemplify these two models trough an empirical work.

\section{INDEX}

Mots-clés : contrefactuels, réticence /expression, contrat/milieu

Keywords : Counterfactuals, reticence-expression, contract-milieu

\section{AUTEURS}

\section{GÉRARD SENSEVY}

Professeur des universités, Université de Bretagne Occidentale, CREAD Université Rennes 2

\section{NATHALIE VIGOT}

Maître formatrice et docteure, Université de Bretagne Occidentale, CREAD Université Rennes 2 